\title{
On the elimination of infinities in the PO component of equivalent edge currents
}

\author{
Taner Oguzer, Ayhan Altıntaş \\ Department of Electrical and Electronics Engineering, Bilkent University, 06533 Bilkent, Ankara. Turkey \\ O. Merih Büyükdura \\ Department of Electrical and Electronics Engineering. Middle East Technical University, 06531, Ankara, Turkey
}

Received 31 October 1991, Revised 3 September 1992

\begin{abstract}
Equivalent edge currents for the Physical Optics (PO) contribution to the edge diffraction are derived by choosing a proper coordinate system for a half plane. It is seen that these expressions for the PO component are free from singularities except at shadow and reflection boundaries on the Keller Cone. To find the total diffracted field, the expressions for the fringe contribution given earlier by Michaeli should be used along with the PO contribution obtained here. The procedure is applied to the problems of backscattering from square and triangular plates. Higher order diffractions are also accounted for by combining the equivalent currents with the Uniform Theory of Diffraction (UTD). The results obtained are compared with previous solutions and measured data.
\end{abstract}

\section{Introduction}

It is known that the Geometrical Theory of Diffraction (GTD) [1] and its uniform versions the Uniform Theory of Diffraction (UTD) [2] and the Uniform Asymptotic Theory (UAT) [3] fail in the vicinity of diffracted ray caustics. To overcome this difficulty, an equivalent current method (ECM) has been suggested by Ryan and Peters [4]. In this method, equivalent electric and magnetic currents which are considered to radiate in free space are introduced in the place of the diffracting edge. These currents are heuristically introduced in such a manner that when their radiation integral is evaluated asymptotically, the leading term reduces to the GTD diffracted field away from caustic regions.

Since these equivalent currents are derived from the GTD fields; strictly speaking, they are valid for observation directions only on the Keller Cone of diffraction. A generalization of this method has been proposed by Knott and Senior [5] which is based on reciprocity considerations. More rigorous asymptotic expressions for equivalent currents for arbitrary directions of observation have been achieved independently by Mitzner [6] and Michaeli [7] based on identifying the equivalent current line integral with the asymptotic edge contribution to the radiation integral of the physical surface current density on a conducting half-plane. Unfortunately, these expressions for the equivalent currents become infinite at various angles of observation. In a subsequent paper [8], Michaeli considered these currents as arising separately from physical optics (PO) and fringe components and showed that for the fringe component the infinities can be eliminated by choosing a proper skew coordinate system for the asymptotic integration. However, it was asserted by the same author [9] that the infinities in the PO component cannot be eliminated in a similar way. But, again in [9], a different procedure is proposed to obtain the uniform PO equivalent edge currents by using the Fresnel functions.

In the present paper, equivalent edge currents for the PO contribution are derived by choosing a proper coordinate 
system for a half plane in a manner similar to the treatment in [8]. It is seen that these expressions for the PO component are free from singularities except at shadow and reflection boundaries on the Keller Cone. To find the total scattered field, the expressions for the fringe component given in [8] should be used along with the PO contribution given here.

The procedure proposed here is applied to the problems of backscattering from square and triangular plates. Higher order diffractions are also accounted for by combining the equivalent currents with the UTD. The results obtained are compared with previous solutions and measured data.

\section{Equivalent edge currents for far zone scattering}

Consider a perfectly conducting flat surface $S$ on the $x y$-plane with an edge $C$ as shown in Fig. 1 . At any point on the edge, the unit vector $\hat{i}$ is tangent to the edge, $\hat{n}$ is normal to the surface, and the unit binormal vector is given by

$$
\hat{b}=\hat{n} \times \hat{t} .
$$

The angles $\phi, \phi^{\prime}, \beta$ and $\beta^{\prime}$ are measured as shown in Figs. 1 and $2 . R$ is the distance to the observation point measured from the origin at the coordinate system. $\hat{s}^{\prime}$ and $\hat{s}$ are the unit vectors for incident and observation directions. The scattered far field from this structure is given by the following radiation integral

$$
\hat{\mathbf{E}}^{\mathrm{s}}=\frac{\mathrm{j} k Z}{4 \pi} \frac{\mathrm{e}^{\mathrm{j} k R}}{R} \hat{s} \times \hat{s} \times \iint_{\mathrm{S}} \hat{J}^{\mathrm{T}}(x, y) \mathrm{e}^{\mathrm{j} k(\hat{x}+\hat{y}) \cdot s} \mathrm{~d} s,
$$

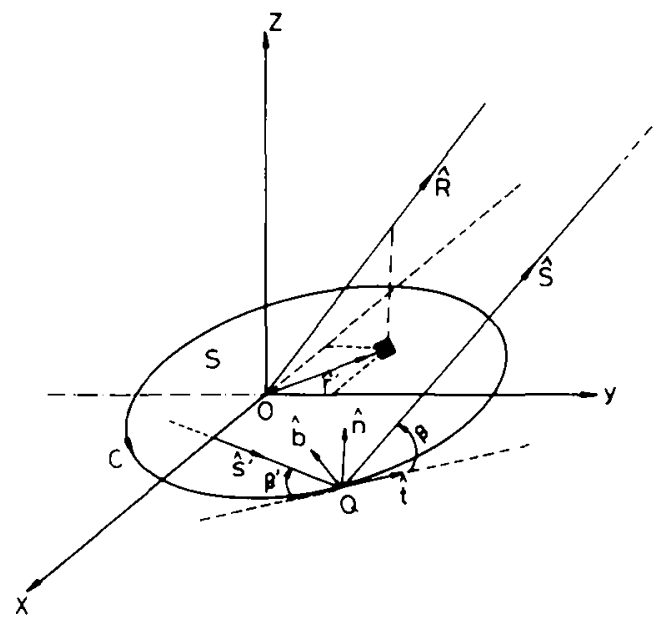

Fig. 1. Perfectly conducting flat plate

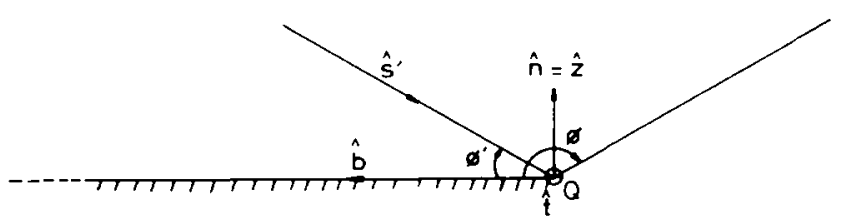

Fig. 2. Two dimensional cross-section in $\hat{n}-\hat{b}$-plane at the diffraction point $Q$. 
where $Z$ is the intrinsic impedance, $k$ is the wavenumber, and $\hat{J}^{\mathrm{T}}(x, y)$ is the total induced current on the surface.

By using the asymptotic evaluation approach in [7], the edge diffracted part of the total scattered far field written in the $(t, b)$ coordinate system is given by

$$
\hat{E}^{\mathrm{d}}=\frac{\mathrm{j} k Z}{4 \pi} \frac{\mathrm{e}^{-\mathrm{j} k R}}{R} \hat{s} \times \hat{s} \times \int_{C} \vec{K}(t) \mathrm{e}^{\mathrm{j} k t i \cdot \hat{\mathrm{d}} t} .
$$

where

$$
\vec{K}(t)=\int_{0} \hat{J}^{\mathrm{T}}(t, b) \mathrm{e}^{\mathrm{j} k b \hat{b} \cdot \hat{s}} \mathrm{~d} b,
$$

and " $o$ " denotes the asymptotic end-point contribution at $b=0$.

The edge diffracted field can also be expressed as due to the equivalent edge currents along the edge:

$$
\hat{E}^{\mathrm{d}}=\frac{\mathrm{j} k Z}{4 \pi} \frac{\mathrm{e}^{-\mathrm{j} k R}}{R} \int_{C}[\hat{s} \times \hat{s} \times \hat{\imath} \mathbf{I}+Y \hat{s} \times \hat{t} \mathbf{M}] \mathrm{e}^{\mathrm{j} k \hat{\imath} \cdot \hat{s}} \mathrm{~d} t .
$$

Equating the two integrands, the following equivalent edge currents

$$
\begin{aligned}
& \mathbf{I}=\frac{1}{\sin ^{2} \beta} \hat{s} \cdot[(\hat{t} \times \hat{s}) \times \vec{K}], \\
& \mathbf{M}=\frac{Z}{\sin ^{2} \beta} \hat{i} \cdot(\hat{s} \times \vec{K}),
\end{aligned}
$$

are obtained after some manipulation.

The determination of equivalent currents $\mathbf{I}$ and $\mathbf{M}$ requires the asymptotic evaluation of the integral of the surface current $J^{\mathrm{T}}$ to obtain $\vec{K}(t)$ in (4). The problem of the scattering from a half-plane will be examined as the canonical problem for this purpose. The total induced surface current $J^{\mathrm{T}}$ is decomposed into the $J^{\mathrm{GO}}$ (geometrical optics) and $J^{\mathrm{f}}$ (the fringe or the "non-uniform" component as named by Ufimtsev [10]). We will examine the radiation from each component separately.

\section{Fringe component of equivalent edge currents}

The derivation of the fringe component of the equivalent edge currents is obtained earlier by Michaeli [8]. For the sake of completeness, that derivation is summarized here and it requires the asymptotic end point evaluation of the following integral:

$$
\vec{K}^{f}(z)=\int_{0} \vec{J}^{f}(x, z) \mathrm{e}^{\mathrm{j} k x \dot{x} \cdot \hat{\mathrm{d}} \mathrm{d} x} .
$$

The fringe current $\vec{J}^{\mathrm{f}}(x, z)$ cannot be represented near the edge $(x=0)$ in a simple amplitude-phase form. However, away from the edge, the GTD gives a phase variation of $-k x \hat{x} \cdot \hat{\sigma}$. This phase describes $\vec{J}^{f}(x, z)$ as a wave propagating in the $\hat{\sigma}$ direction which is the direction of diffracted rays at the intersection of the Keller Cone with the half-plane. If it is assumed that the ray behaviour of the fringe surface field is valid up to the edge, then the integral in (20) becomes infinite, when

$$
\hat{s} \cdot \hat{x}=\hat{\sigma} \cdot \hat{x} .
$$


In other words, the singularity condition is satisfied when the phase of $\vec{J}^{\dagger}(x, z)$ cancels the phase of the exponent in the integral. This singularity condition represents a cone around the $x$ axis. With this argument, Michaeli [8] states that these expected singularities can be reduced to a single direction by choosing a proper skew coordinate system. Therefore $\sigma$ and $z$ coordinates are selected for the evaluation of the surface integral instead of the Cartesian coordinates $x$ and $z$ with the following replacement:

$$
\begin{aligned}
& x \rightarrow \sigma \sin \beta^{\prime} . \\
& z \rightarrow z+\sigma \sin \beta^{\prime} .
\end{aligned}
$$

Using these coordinates, the integral to be evaluated asymptotically becomes

$$
\vec{K}^{\prime}(z)=\sin \beta^{\prime} \int_{o} \vec{J}^{\prime}\left(\sigma \sin \beta^{\prime}, z+\sigma \cos \beta^{\prime}\right) \mathrm{e}^{\mathrm{j} k \sigma \dot{\sigma} \cdot s} \mathrm{~d} \sigma .
$$

This asymptotic evaluation is done and the resultant expressions are given in $|8|$.

\section{PO component of equivalent edge currents}

The determination of $\mathrm{PO}$ component of equivalent edge currents requires the asymptotic evaluation of the following integral:

$$
\vec{K}^{\mathrm{PO}}(z)=\int_{0} \vec{J}^{(i)}(x, z) \mathrm{e}^{j k x i \cdot \hat{d}} \mathrm{~d} x
$$

Here, the phase of $\vec{J}^{\mathrm{GO}}(x, z)$ is $-k x \hat{x} \cdot \hat{s}^{\prime}$. Hence, the singularity condition of the integral becomes

$$
\hat{x} \cdot \hat{s}=\hat{x} \cdot \hat{s}^{\prime}
$$

The replacement of the $\hat{x}$ direction by the $\hat{\sigma}$ direction for the integration given in the previous section does not have a similar effect on the PO components. Because, as mentioned in 18], such a step "merely replaces the previous cone of singular $\hat{s}$-directions, by another cone, defined by $\hat{s} \cdot \hat{\sigma}=\hat{s}^{\prime} \cdot \hat{\sigma}$, and only for glancing incidence, $\hat{\sigma}=\hat{s}^{\prime}$, does the latter collapse into a single direction, $\hat{s}=\hat{\sigma}^{\prime}$. However, the above argument assumes a skew coordinate direction fixed by the incidence angle for all observation directions. In fact, for equivalent currents, there is no need for such a restriction; the skew coordinate direction may be determined by both the incident and observation directions $\left(\hat{s}^{\prime}\right.$ and $\left.\hat{s}\right)$. After all, the expressions for the equivalent edge currents are always functions of observation aspect. So for each observation direction, the skew coordinate direction on the surface of the half-plane is determined separately. To apply this approach, we will again use the skew coordinate system $(\sigma, z)$ with an arbitrary skewness angle $\gamma$ as follows:

$$
\begin{aligned}
& x \rightarrow \sigma \sin \gamma, \\
& z \rightarrow z+\sigma \cos \gamma .
\end{aligned}
$$

Then, the end-point contribution from the integral

$$
\vec{K}^{\mathrm{PO}}(z)=\sin \gamma \int_{\sigma} \vec{J}^{\mathrm{GO} O}\left(\sigma \sin \gamma_{\cdot} z+\sigma \cos \gamma\right) \mathrm{e}^{\mathrm{j} k \sigma \dot{\sigma} \cdot \hat{\mathrm{d}} \sigma},
$$


is evaluated as

$$
\begin{aligned}
K_{x}^{\mathrm{PO}}= & 2 Y \sin \beta^{\prime}-\frac{\sin \gamma}{j k\left[\left(\sin \beta^{\prime} \cos \phi^{\prime}+\sin \beta \cos \phi\right) \sin \gamma+\cos \gamma\left(\cos \beta-\cos \beta^{\prime}\right)\right]}, \\
K_{z}^{\mathrm{PO}}= & -\frac{2 Y \cos \phi^{\prime} \cos \beta^{\prime} \sin \gamma}{\left(\sin \beta^{\prime} \cos \phi^{\prime}+\sin \beta \cos \phi\right) \sin \gamma+\cos \gamma\left(\cos \beta-\cos \beta^{\prime}\right)} \\
& +\frac{2 Y \sin \phi^{\prime} \sin \gamma}{\left(\sin \beta^{\prime} \cos \phi^{\prime}+\sin \beta \cos \phi\right) \sin \gamma+\cos \gamma\left(\cos \beta-\cos \beta^{\prime}\right)} .
\end{aligned}
$$

The PO equivalent edge currents are then obtained as:

$$
\begin{aligned}
\mathbf{I}^{\mathrm{PO}}= & -\frac{2 Y}{\mathrm{j} k} E_{z}^{\mathrm{i}} \frac{\sin \phi^{\prime} \sin \gamma}{\sin \beta^{\prime}\left[\left(\sin \beta^{\prime} \cos \phi^{\prime}+\sin \beta \cos \phi\right) \sin \gamma+\cos \gamma\left(\cos \beta-\cos \beta^{\prime}\right)\right]} \\
& +\frac{2}{\mathrm{j} k} H_{z}^{\mathrm{i}} \sin \gamma \frac{\cot \beta^{\prime} \cos \phi^{\prime}+\cot \beta \cos \phi}{\left[\left(\sin \beta^{\prime} \cos \phi^{\prime}+\sin \beta \cos \phi\right) \sin \gamma+\cos \gamma\left(\cos \beta-\cos \beta^{\prime}\right)\right]}, \\
\mathbf{M}^{\mathrm{PO}}= & \frac{2 Z}{\mathrm{j} k} H_{z}^{\mathrm{i}} \frac{\sin \phi \sin \gamma}{\sin \beta\left[\left(\sin \beta^{\prime} \cos \phi^{\prime}+\sin \beta \cos \phi\right) \sin \gamma+\cos \gamma\left(\cos \beta-\cos \beta^{\prime}\right)\right]} .
\end{aligned}
$$

It is seen that these expressions are functions of the skewness angle $\gamma$. Hence they are nonunique.

If the expressions in this and the previous section are examined on the Keller Cone, i.e., for $\beta^{\prime}=\beta$, it is observed that

$$
\mathbf{I}^{\mathrm{f}}\left(\beta=\beta^{\prime}\right)+\mathbf{I}^{\mathrm{PO}}\left(\beta=\beta^{\prime}\right)=\mathbf{I}^{\mathrm{GTD}}
$$

and

$$
\mathbf{M}^{\mathrm{f}}\left(\beta=\beta^{\prime}\right)+\mathbf{M}^{\mathrm{PO}}\left(\beta=\beta^{\prime}\right)=\mathbf{M}^{\mathrm{GTD}}
$$

where $I^{\text {GTD }}$ and $\mathbf{M}^{\text {GTD }}$ are the equivalent currents suggested in [4]. Hence, it is observed that when the radiation integral of the currents suggested in this work are evaluated asymptotically, they yield the GTD diffracted fields, as expected. This result suggests that, on the Keller Cone, the PO equivalent currents given in this section should be independent of the skewness angle $\gamma$. Indeed, for $\beta^{\prime}=\beta$, we have

$$
\begin{aligned}
& \mathbf{I}^{\mathrm{PO}}\left(\beta=\beta^{\prime}\right)=\frac{2 Y}{\mathrm{j} k} E_{z}^{\mathrm{i}} \frac{\sin \phi^{\prime}}{\sin ^{2} \beta\left(\cos \phi+\cos \phi^{\prime}\right)}+\frac{2}{\mathrm{j} k} H_{z}^{\mathrm{i}} \frac{\cot \beta}{\sin \beta} \\
& \mathbf{M}^{\mathrm{PO}}\left(\beta=\beta^{\prime}\right)=\frac{2}{\mathrm{j} k Y} H_{z}^{\mathrm{i}} \frac{\sin \phi}{\sin ^{2} \beta\left(\cos \phi+\cos \phi^{\prime}\right)} .
\end{aligned}
$$

These expressions have no singularities except at the incidence and reflection boundaries. But, for an arbitrary choice of $\gamma$, the equivalent currents may have other singularities off the Keller Cone. With this in mind, we let $\gamma$ be

$$
\cot \gamma=\frac{\cos \beta-\cos \beta^{\prime}}{\sin \beta^{\prime} \cos \phi^{\prime}+\sin \beta \cos \phi}
$$

to obtain the PO equivalent currents as follows: 


$$
\begin{aligned}
\mathbf{I}^{(\mathrm{C})}= & -\frac{2 \gamma}{\mathrm{j} k} E_{:}^{\mathrm{i}}=\frac{\sin \phi^{\prime}\left(\sin \beta \cos \phi+\sin \beta^{\prime} \cos \phi^{\prime}\right)}{\sin \beta^{\prime}\left[\left(\cos \beta-\cos \beta^{\prime}\right)^{2}+\left(\sin \beta \cos \phi+\sin \beta^{\prime} \cos \phi^{\prime}\right)^{2}\right]} \\
& +\frac{2}{\mathrm{j} k} H_{:}^{\prime} \frac{\left(\sin \beta \cos \phi+\sin \beta^{\prime} \cos \phi^{\prime}\right)\left(\cot \beta \cos \phi+\cot \beta^{\prime} \cos \phi^{\prime}\right)}{\left[\left(\cos \beta-\cos \beta^{\prime}\right)^{2}+\left(\sin \beta \cos \phi+\sin \beta^{\prime} \cos \phi^{\prime}\right)^{2}\right]} \\
\mathbf{M}^{\mathrm{PO})}= & \frac{2 Z}{\mathrm{j} k} H_{:}^{\mathrm{i}}=\frac{\sin \phi\left(\sin \beta \cos \phi+\sin \beta^{\prime} \cos \phi^{\prime}\right)}{\left[\left(\cos \beta-\cos \beta^{\prime}\right)^{2}+\left(\sin \beta \cos \phi+\sin \beta^{\prime} \cos \phi^{\prime}\right)^{2}\right]} .
\end{aligned}
$$

The only singularities of these expressions occur at the following aspects:

$$
\phi \mp \phi^{\prime}=\pi
$$

and

$$
\beta=\beta^{\prime}
$$

which represent the incidence and reflection boundaries on the Keller Cone. Physically, the direction of the skew coordinate $\hat{\sigma}$ in this case is the direction of the projection of $\left(\hat{s}-\hat{s}^{\prime}\right)$ vector onto the half-plane. It is a function of both $\hat{s}^{\prime}$ and $\hat{s}$, as described earlier. The natural question to ask at this point is what happens when $\left(\hat{s}-\hat{s}^{\prime}\right)$ vector has zero projection on the half-plane; in other words, when $\left(\hat{s}-\hat{s}^{\prime}\right)$ has only a normal component to the surface of the half-plane. In this case, the above definition of $\hat{\sigma}$ fails. However, a close examination reveals that when $\left(\hat{s}-\hat{s}^{\prime}\right)$ vector has only a normal component to the half-plane, the observation direction is either on the incident or on the reflection shadow boundary on the Keller Cone. Both of these cases correspond to directions for which the field is non ray optical and the equivalent current concept is not valid.

The PO equivalent current expressions for arbitrary skewness angle in (20) and (21) can be obtained asymptotically from the expressions of Michaeli [9].

It is also noted that the equivalent current expressions given in (27) and (28) are the same as those in [11,12] where they are determined for a finite size plate by an application of Stokes' Theorem.

\section{Numerical results}

To check the accuracy of the derived equivalent currents, we applied them to the problem of backscattering from square and triangular plates. In both problems, the $x z$-plane is the observation plane and the radar cross section (RCS) is calculated as a function of the elevation angle $\theta$ from the $z$-axis for both $E$ - and $H$-plane configurations. In the $E$-plane, the incident and backscattered fields have an electric field parallel to the plane of observation $(\hat{\theta}$ component ) and in the $H$-plane, the incident and backscattered electric field have an electric field perpendicular to the plane of observation ( $\hat{\phi}$ component). In the calculations, second order diffractions are also included in which UTD diffraction coefficients are used to find the first order diffractions. The regions of illumination are determined by using the geometrical arguments.

\subsection{Square plate}

The square plate shown in Fig. 3 is parallel to the $x y$-plane. Our results for $E$ - and $H$-plane are compared with the solution in [13]. As seen in Fig. 4, for $E$-plane calculations, there is very little difference between the present result and that in [13]. The results are also close to the experimental data of Ross [14]. In the $H$-plane illumination, the agreement between our result and that in [13] is good and they coincide with the experimental results away from 


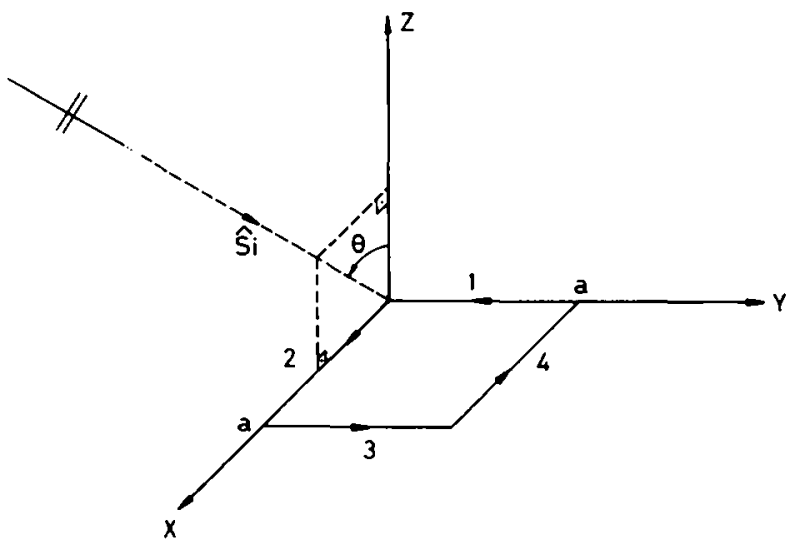

Fig. 3. Perfectly conducting square plate.

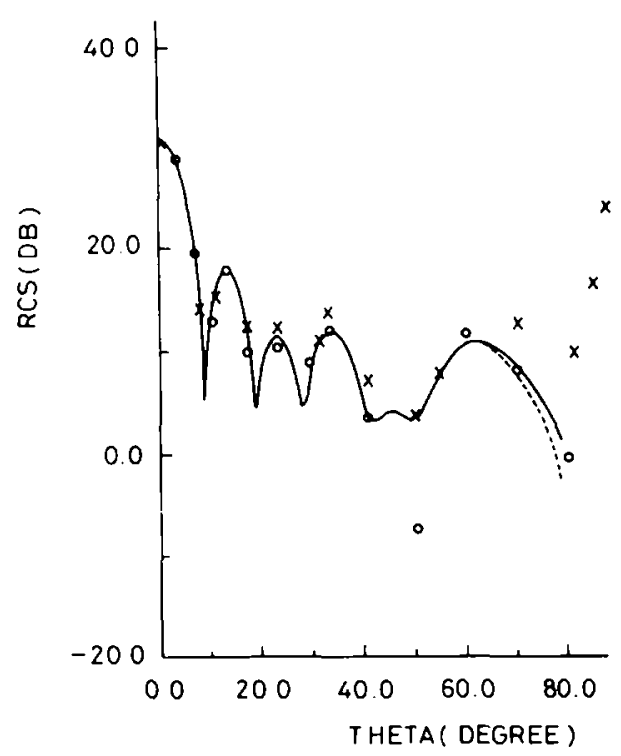

- Calculated

oo Measurement of Ross [14]

--- Sikta

$x \times$ Calculation of Ross

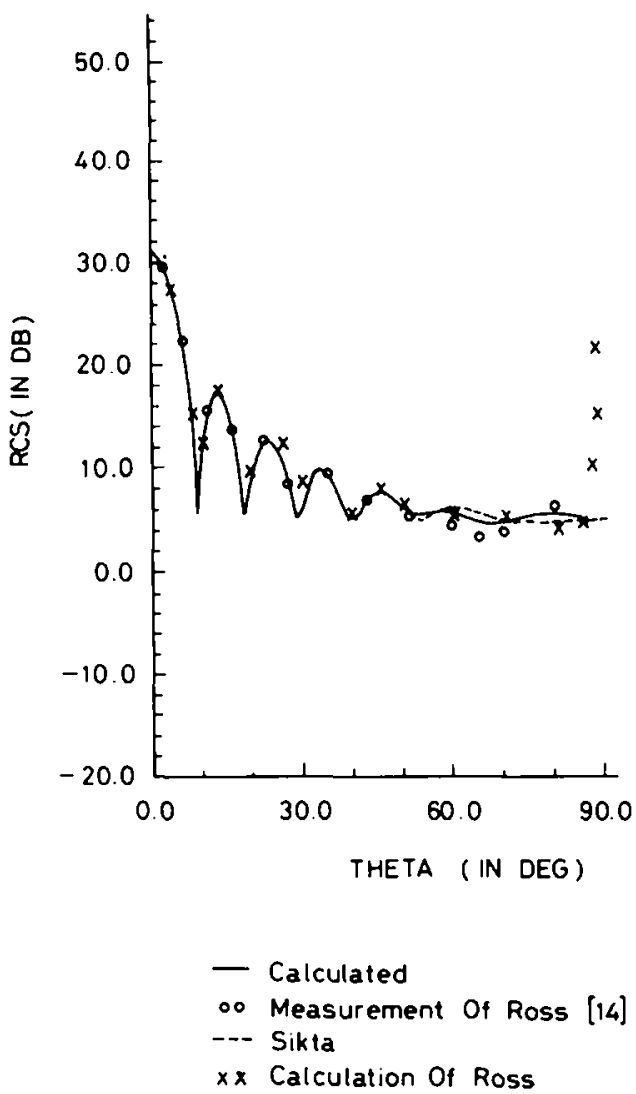

Fig. 4. Backscattering from the square plate of Fig. 3: $E$-polarization $(a=3.125 \lambda)$.

Fig. 5. Backscattering from the square plate of Fig. 3: $H$-polarization $(a=3.125 \lambda)$.

the grazing incidence region ( see Fig. 5). By including the second order currents in the analysis, we were able to predict the small oscillations for large elevation angles. 


\subsection{Triangular plate}

The triangular plate is shown in Fig. 6 and it is parallel to the $y z$-plane. Previous results were obtained by Sikta in [13] and Michaeli in [15]. Sikta used the GTD equivalent currents including the second and third order interactions, corner diffraction and the edge wave mechanism in his analysis. Later on. Michaeli analyzed the same problem by including the first and second order fringe equivalent currents and the PO contribution left as a surface integral. We used the fringe and PO equivalent currents with the second order diffractions combining the equivalent currents with the UTD. Our $E$-plane and $H$-plane calculations are compared in Figs. 7 and 8 with Sikta's solution and the measurements. Both present and Sikta's results are fairly accurate around the broadside direction $(45<\theta<120)$ and our results have a better prediction of the variation of the pattern for $45<\theta<90$. Outside the broadside direction, both results deviate from the experiments. Although it is not shown here, Michaeli's solution is similar in nature, but in general closer to experimental results than both Sikta's and our result.

\section{Conclusions}

In this paper, we have obtained PO equivalent currents in the same way as fringe equivalent currents by choosing a proper oblique direction for the asymptotic integration. This work therefore complements the work of Michaeli

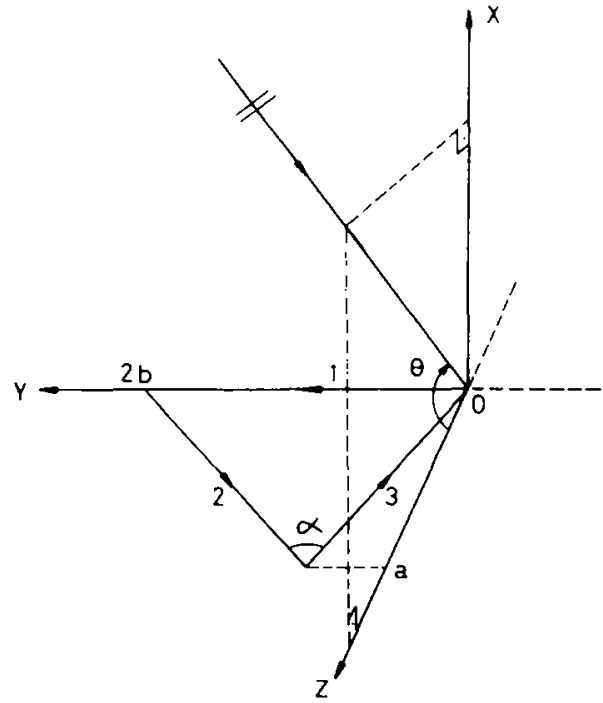

Fig. 6. Perfectly conducting triangular plate.

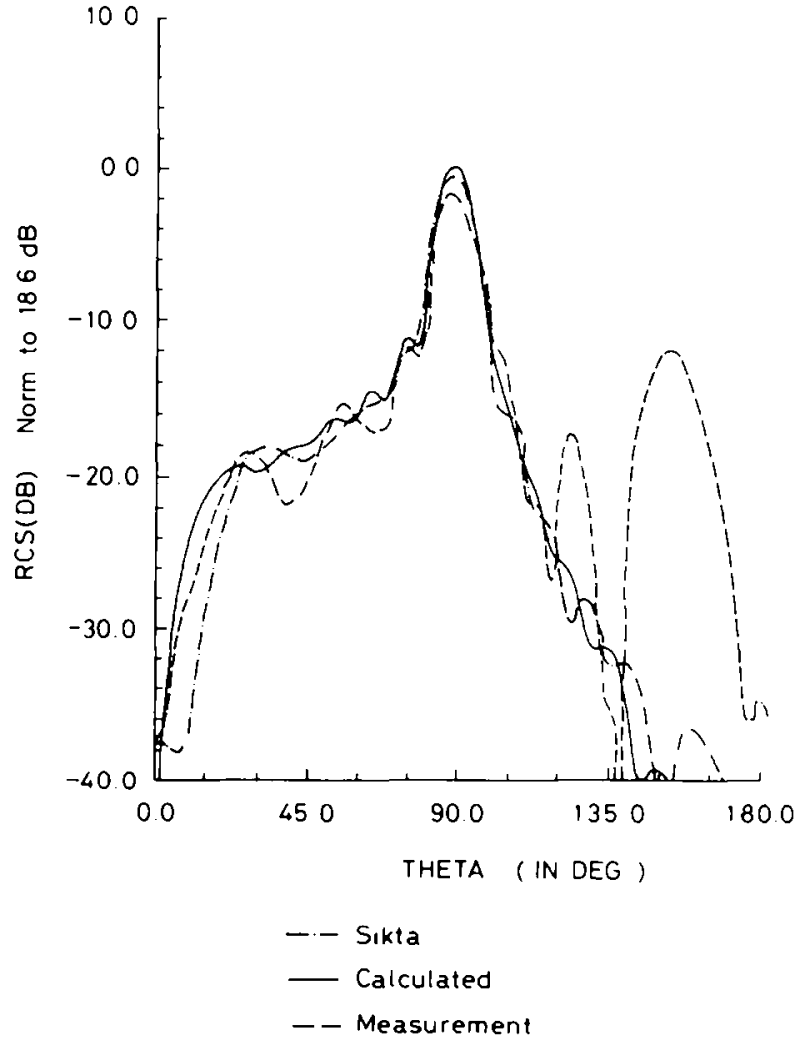

Fig. 7. Backscattering from the triangular plate of Fig. 6: $E$-polarization $(a=3 \lambda, \alpha=30 \mathrm{deg})$. 


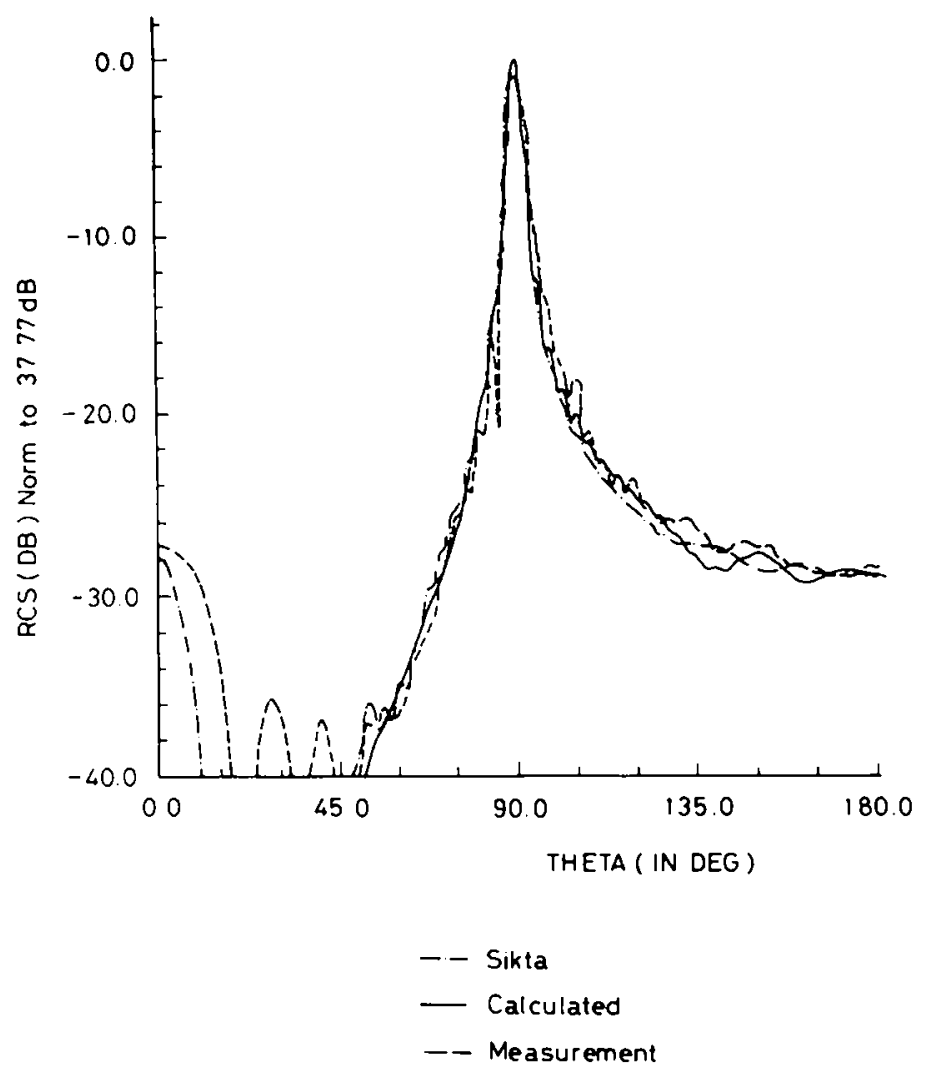

Fig. 8. Backscattering from the triangular plate of Fig. 6: $H$-polarization $(a=9 \lambda, \alpha=30 \mathrm{deg})$.

18]. The PO and fringe equivalent currents are valid for arbitrary directions of observation, so they are more general than the GTD equivalent currents.

The applications of these equivalent currents have been illustrated on two examples of backscattering from square and triangular plates.

\section{References}

[1] J.B. Keller, "Geometrical theory of diffraction", J. Opt. Soc. Am. 52, 116-130 (1962).

[2] R.G. Kouyoumjian and P.H. Pathak, "A uniform geometrical theory of diffraction for an edge in a perfectly conducting surface", Proc. IEEE 62. 1448-1461 (1974).

[3] S.W. Lee and G.A. Deschamps, "A uniform asymptotic theory of EM diffraction by a curved wedge", IEEE Trans. AP-24, 25-34 (1976).

[4] C.E. Ryan and L. Peters, "Evaluation of edge diffracted fields including equivalent currents for the caustic regions", IEEE Trans. AP.17, 292-299 (1960).

[5] E.F. Knott and T.B.A. Senior, "Comparison of three high-frequency diffraction techniques", Proc. IEEE 62, 1468-1474 (1974).

[6] K.M. Mitzner, "Incremental length diffraction coefficients". Aircraft Division. Northrop Corp., Tech. Rep. AFAL-TR-73-296 (1974).

[7] A. Michaeli, "Equivalent edge currents for arbitrary aspects of observation". IEEE Trans. AP-32, 252-258 (1984).

[8] A. Michaeli, "Elimination of infinities in equivalent edge currents, Part I: Fringe current components", IEEE Trans. AP-34, 912-918 (July 1986).

[9] A. Michaeli, "Elimination of infinities in equivalent edge currents, Part Il: Physical optics component" IEEE Trans. AP-34, 1034-1037 (1986). 
[10] P.Ya. Utimtsev, "Method of edge waves in the physical theory of diffraction". Izd-to Sol. Radio, 1-243 (1962).

[11] R.J. Marhefka, O.M. Buyukdura and W. Ebihara. "Radar cross section studies, phase III". Tech. Rep. 716621-1, The Ohio State University. ElectroScience Laboratory, Columbus. Ohio ( 1986 ).

[12] W.B. Gordon. "Far-field approximation to the Kirchoff-Helmholz representations of scattered fields", IEEE Trans. AP-23, 590-592 (1975).

[1.3| F.A. Sikta, "UTD analysis of electromagnetic scattering hy flat plate structures", Ph.D. dissertation. Ohio State Liniversity. Columbus. Ohio ( 1981 ).

[14] R.A. Ross, "Radar cross section of rectangular flat plates as a function of aspect angle", IEEE Trans. AP-14, 329-335 (1966).

[15] A. Michaeli, "Equivalent currents for second order diffraction by the edges of perfectly conducting polygonal surfaces". IEEE Trans. AP-35. 183-190 (1987) 